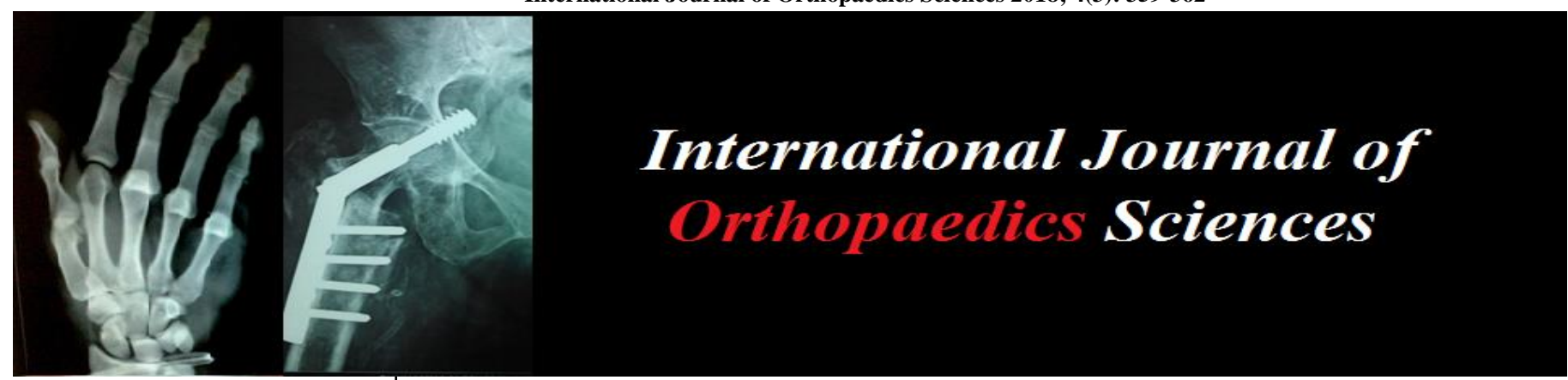

ISSN: $2395-1958$

IJOS 2018; 4(3): 359-362

(C) 2018 IJOS

www.orthopaper.com

Received: 01-05-2018

Accepted: 02-06-2018

Dr. D Vimal Raj

Associate Professor, Department of Orthopaedics, Indira Gandhi

Medical College \& Research

Institute, Puducherry, India
Correspondence

Dr. D Vimal Raj

Associate Professor, Department

of Orthopaedics, Indira Gandhi

Medical College \& Research

Institute, Puducherry, India

\section{Functional and radiological outcome of pertrochanteric fractures treated with proximal femoral nailing in post- menopausal women, age $>60$ years}

\section{Dr. D Vimal Raj}

DOI: https://doi.org/10.22271/ortho.2018.v4.i3g.61

\begin{abstract}
Pertrochanteric fractures (intertrochanteric and subtrochanteric) are one of the common fractures encountered in general population especially with post-menopausal women following a fall due to high energy trauma requiring immediate and active intervention. The main benefit of Proximal Femoral Nailing is being a closed technique offering an excellent reduction at the fracture site and adequate strength for weight bearing even in unstable hip fractures. This intramedullary device is proven to be biomechanically superior to DHS which is an extra medullary device in the treatment of Pertrochanteric fractures. The objective of this study is to analyse and evaluate the functional and radiological outcome of Pertrochanteric fracture treated with proximal femoral nail. A study population was selected and was carried out with a total of 30 patients (post-menopausal) treated with proximal femoral nail from the year 2015-2017. All the patients were selected based on inclusion criteria such as closed fractures of less than 3 weeks and age above 60 years. The appropriate selected patients were assessed both clinically and radiologically at regular intervals of $4 \mathrm{wks}, 8 \mathrm{wks}, 12 \mathrm{wks}, 16 \mathrm{wks}$ and $20 \mathrm{wks}$. The functional outcome was assessed using Harris Hip score. From our selected study sample we found majority of the patients were unstable fractures (70\%) and others were stable fractures $(30 \%)$. Based on the union, $50 \%$ of patients showed full union by 12 to 15 weeks and $35 \%$ showed full union by 17 to 20 weeks. We had $85 \%$ of the patients with good anatomical results and $50 \%$ of the patients with excellent functional results. PFN is a quite safe, well tolerated and effective instrumentation useful in the treatment of all Pertrochanteric fractures. Being a closed intramedullary procedure early mobilization and rehabilitation is possible with early restoration of premorbid functions.
\end{abstract}

Keywords: Pertrochanteric, proximal femoral nailing, femur, post-menopausal

\section{Introduction}

Pertrochanteric fractures are one of the common fractures encountered in general population especially in post-menopausal women as a result of simple fall and after high velocity injury involving both direct and indirect forces. Direct forces travels along the axis of the femur or the greater trochanter leading to Pertrochanteric fracture. PFN is the latest intramedullary device designed by AO / ASIF in 1996 consisting two proximal screws for the neck and head and two distal locking screws. The key feature is being a closed technique having good reduction at the fracture site and provides sufficient strength to allow weight bearing even in unstable hip fractures. This intramedullary device is proven to be biomechanically superior to DHS which is an extra medullary device in the treatment of Pertrochanteric fractures.

\section{Materials and Methods}

The study was conducted with a sample size of 30 patients with Pertrochanteric fractures admitted @ Pondy Surgical Centre from 2015-2017.The inclusion criteria such as patients with Pertrochanteric fractures of less than 3 weeks, Closed fractures and women age above 60 and the exclusion criteria such as Fractures of more than 3 weeks, Open fractures, Age below 60 years were involved. After clinical assessment, blood investigation and radiological work up was done and pre Anaesthetic check up obtained. All the patients underwent Proximal Femoral Nailing as proposed. Type of surgery and the application of technique used for fixation of the implant was documented. 
Post-operative care such as use of medications, regular wound inspection, suture removal and mobilization from day 1 with gait training was initiated using a walker or crutches were also documented. Patients were advised for regular follow ups on OPD basis at 4, 8, 12, 16, 20 weeks post operatively. During each visit, patients were assessed both clinically and radiologically based on severity of, hip range of movements, fracture union and complications using Harris Hip score.

\subsection{Operative Technique}

Under spinal or general anaesthesia patient on supine was then placed on the fracture table with his contralateral leg placed on a leg support. 2 to $3 \mathrm{~cm}$ incision was made $2 \mathrm{~cm}$ above proximal end of the greater trochanter on the extension line of the femoral axis. Soft tissues were separated and the trochanter tip was reached, the entry point was felt which is located at the junction of anterior two third and posterior two thirds of greater trochanter. The entry was made with the awl and the guide wire was passed along the femoral axis and the reduction was checked with $\mathrm{C}$ arm in two planes. The proximal and distal two fragments were reamed using 10, I 1, $12,13,14$ reamers. Nail of at least $2 \mathrm{~mm}$ less than that of the last reamer used was selected. After fixing into the jig using the AP view, the PFN was inserted by hand until the lag screw holes are lined up within the centre of femoral neck. Later the guide sleeve was introduced through the jig and a $2 \mathrm{~cm}$ incision was made on the lateral aspect of the thigh. A $2.8 \mathrm{~mm}$ guide wire was inserted through the drill sleeve and position were checked under image intensifier in AP and lateral view. A position in the caudal area of the femoral head
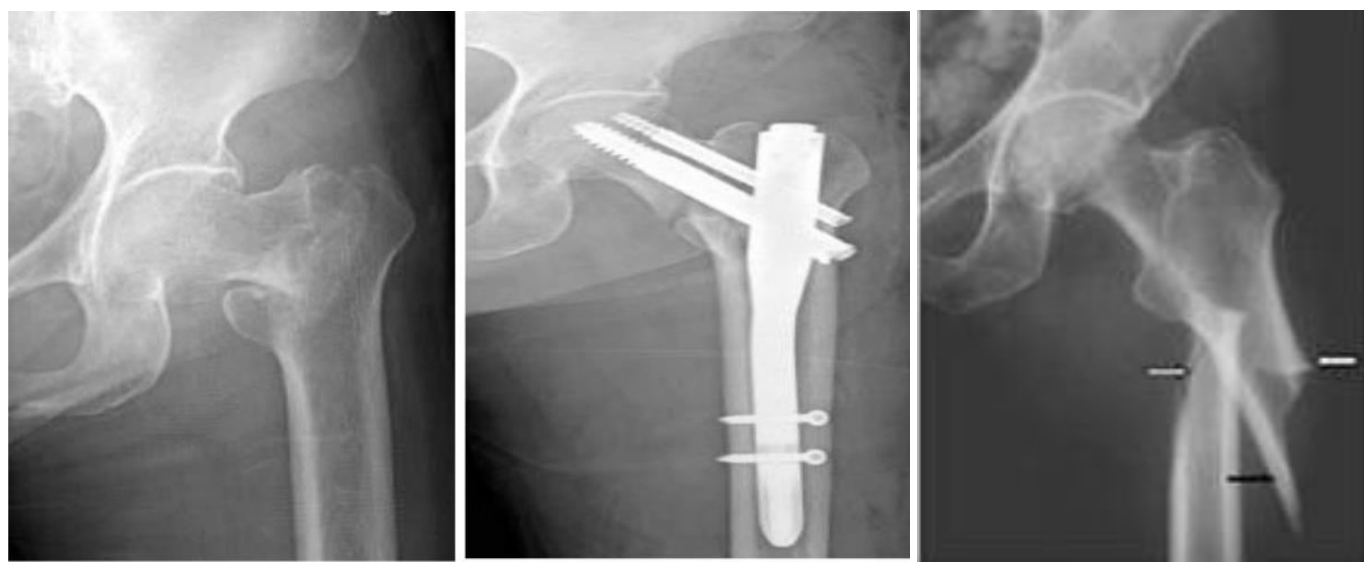

\subsection{Discussion}

The femur is the longest and strongest bone of the body and like all long bones consists of a shaft and two ends. It articulates at its upper end with the hipbone and at its lower end with both the patella and the tibia. The upper end of the femur comprises a head, a neck, a greater and a lesser trochanter. The neck of the femur is rather more than half a Sphere \& and is directed upwards, medially and slightly anteriorly. The neck is about $5 \mathrm{~cm}$ long, connects the head to the shaft. Anteriorly at the junction of the shaft and the neck is a rough bony ridge, the intertrochanteric line and the region just below that is the subtrochanteric area. Posteriorly a prominent ridge of bone, the intertrochanteric crest is a round protuberance called the quadrate tubercle. The greater trochanter is large and quadrangular laterally positioned and irregular. The upper posterior margin overhangs the trochanteric fossa. The greater trochanter provides insertion for most of the muscles of the gluteal region. The lesser trochanter is a conical eminence, which projects medially and backwards from the shaft at its junction with the lower and

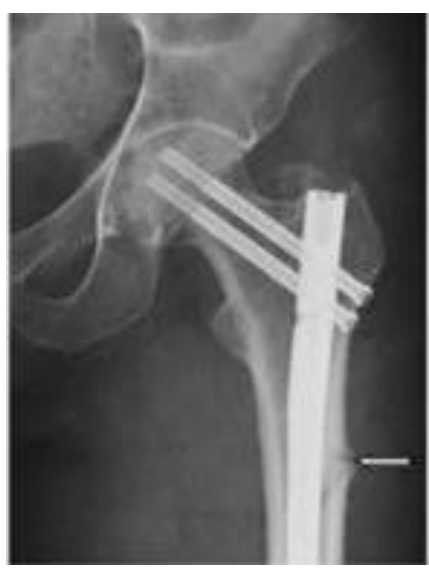

was selected so that both proximal screws can be inserted. In lateral view, the wire should be in the centre the femoral neck. The guide wire was inserted subchondrally or $5 \mathrm{~mm}$ away into the femoral head. After reaming over the guide wire, the appropriate caudal hip screw was introduced. Then the cranial hip screw was introduced just above the caudal hip screw using the jig. Both the distal locking screws were inserted to improve the stability and the wound is closed with 1-3 sutures.

\section{Results and Discussion \\ 3.1 Results}

A Prospective study was conducted on 25 patients who underwent closed reduction internal fixation with proximal Femoral nail to study the functional outcome based on the type of surgery.Age of involvement women more than 60 years of age who had a slip and fall $(80 \%)$ and the rest were road traffic accidents (20\%). Majority were unstable fractures according to Boyd and Griffin $(70 \%)$ and the rest were stable fractures $(30 \%)$. Post operatively patients were mobilized on the second day with non-weight bearing crutch walking (60\%) and partial weight bearing (45\%) in the first five weeks later with full weight bearing in the first 10 to 14 weeks $(55 \%)$. $50 \%$ of the patients showed full union by 12 to 15 weeks and $35 \%$ showed full union by 17 to 20 weeks, the mean time taken for full union was 16 weeks. The mean range of motion for hip was 101 degrees and the knee was 134 degrees, We had $85 \%$ of the patients with good anatomical results and $50 \%$ of the patients with excellent functional results.

posterior part of the neck. It gives attachment to the psoas major on its summit and the iliacus at its base.

The description of adult vessels is based on the work of Trueta and Harnington (1953). Since the vascular pattern established during the phase of growth is not replaced at maturity, but persists throughout in life, the basic arrangement is one of an epiphyseal and metaphyseal circulation, even when the growth plate has disappeared outline the anastamotic arrangement around the upper femur.

Corck described the blood supply to the proximal end of the femur, which he divided into three major groups. 1) An extra capsular arterial ring located at the base of the femoral neck. 2) Ascending cervical branch of the arterial ring on the surface of the femoral neck. 3) Arteries of the ligamentum teres. The extra capsular arterial ring is formed posteriorly by a large branch of medial femoral circumflex artery and anteriorly by branch from lateral femoral circumflex artery. The ascending cervical branches of retinacular vessels ascend on the surface of the femoral neck in anterior, posterior, medial and lateral groups. The lateral vessels are most 
important. Their proximity to the surface of the femoral neck makes them vulnerable to injury in femoral neck fractures. Blood supply to the femur, like that of all tubular bones, is by the way of metaphyseal, periosteal and endosteal supply. The periosteal supply is related to the multiple muscle origins from the shaft to the femur. The nutrient arteries perforate the femoral shaft along the linea aspera. The arteries are derived from perforating branches of profunda femoris artery. The pertrochanteric and subtrochanteric area can be a site of stress concentration owing to the short radius of curvature at this site. When bone has insufficient opportunity to turnover and remodeling as in metabolic bone disease this may be a site of pathologic fracture. Muscular Forces: The upper end of the femur is surrounded by a mass of powerful muscles. Inclusion of muscles forces necessary during single leg support adds to the complexity of the problem and can increase the stress to much higher values. On the other hand, some muscles such as the tensor fascia lata, may act to partially neutralize bending forces under certain conditions. In a normal hip, the strong gluteal muscles abduct, and the powerful psoas flexes and rotates. These forces are balanced by the abductors and harmstrings. The same muscle forces act upon the fixation device after operation. These force have been shown to generate high forces on the femoral head even when the patient is in bed, which in turn cause stresses in the subtrochanteric area as shown by Koch. Rydell has demonstrated that muscular pull for merely flexing of extending the hip in bed caused as much pressure on the femoral head as did slow walking with or without crutches.

Mechanism of Injury: Pertrochanteric fractures almost invariably occur as a result of a fall, involving both direct and indirect forces. Direct forces act along the axis of the femur or directly over the greater trochanter to result in an intertrochanteric fracture. Indirect forces, including pull of the ilipsoas muscle on the lesser trochanter and the abductors on the greater trochanter, have also been incriminated as a cause of fracture.

Since this fracture is common in older age group, many of these fractures are of fatigue type in which the architecture of the bone is sufficiently inadequate that the fracture takes place with relatively normal activity, carefully taken history in many instances reveal that the patient had some minor mishap, such as turning round or possibly catching a heel, but the fracture often occurs before the fall. In younger individuals with stronger bones the fracture may result from a direct blow or twist in automobile accidents

Subtrochanteric features of the femur are defined as the fracture located in the proximal third of femur from the level of lesser trochanter to a point $5 \mathrm{cms}$ distally. It may occur as an extension from a trochanteric fracture or as an isolated fracture (Boyd and Griffin 1949). fielding defined subtrochanteric area as three inches below the lesser trochanter and fractures occurring in this area are called subtrochanteric fractures.

Mechanism: Fractures of the proximal fetnur occur by one of the three mechanisms, in the elderly, these fractures are usually due to low energy trauma, typically a minor fall. Spiral fractures generally result perhaps with butterfly communication. The second mechanism is that of the trivial trauma, with fracture through a defect in the proximal femur due to neoplasia, most commonly metastatic carcinoma. Such pathological subtrochanteric fractures require assessment and management of the neoplastic process as well as of the fracture. The third mechanism is high-energy trauma, motor vehicle accident or fall from significant height.
Communication, soft tissue damage including possible open wound and presence of associated injuries are typical concerns (Watson, et al). Traumatic Anatomy: Froimson described the pathologic anatomy and physiology in fractures in the subtrochanteric region in which strong opposing muscles immediately distort relationships of the fragments. The proximal fragment is flexed and externally rotated by the iliopsoas muscles, abducted and further rotated externally by the gluteal and the abductor and hamstring groups, shortened by the pull of these muscles and usually posteriorly displaced by the weight of the limb when the patient is in the recumbent position. Biomechanical Studies: During the past century a better understanding of the biomechanics of subtrochanteric fractures has led to the development of better implants and radical changes in treatment modalities. Koch analysed mechanical stress on the femur during weight bearing and found that compression stress exceeds $12001 \mathrm{~b}$ per square inch in the medial subtrochanteric area 1 to $3 \mathrm{cms}$ distal to the lesser trochanter and lateral tensile stress measured about $20 \%$ less. Frankel and Burstein demonstrated significant stress force on the hip and proximal femur with activities such as flexing and extending the hip while supine, indicating continuous stress on the implant system even with bed rest. Rybicke, Simonen, and Weis found that higher forces are generated with eccentrically placed devices, such as plate and devices, compared with centromedullary devices.

PFN for proximal femur is available to fit specific anatomic requirement and fracture type of proximal femur. Proximal locking is achieved through two $8 \mathrm{~mm}$ femoral neck screws. Proximal fixation delivers rotational stability.6* mediolateral implant angle for easy insertion. The anatomic nail design prevents intramedullary stresses and thus secondary fractures at the end of the nail. PFN nail sizes are 9, 10, 11 , \&amp; $12 \mathrm{~mm}$ lengths of $20 \mathrm{~cm}, 22 \mathrm{~cm}$, \&amp; $24 \mathrm{~cm}$. Cervical screws are of $8 \mathrm{~mm}$ cannulated with lengths varying from 70 to $110 \mathrm{~mm}$, Distal locking with two $5 \mathrm{~mm}$ screws. PFN for pertrochantric fractures is a versatile implant system which is used in both simple and complex fractures in the pertrochantric region. PFN consists of 3 components.1. The intramedullary nail.2. The cervical screws3. The distal locking screws. The cervical screws transmit the forces acting on the proximal femur to the intramedullary nail. Early load bearing of the fracture is possible. Two proximal screws ensure rotational stability of the PFN during the operation and subsequent healing process. The PFN is tapered at the diatal end. This reduces stiffness and stress peaks, which can cause pain. Holes in the distal part allow a choice of either static or dynamic locking.

\section{Conclusion}

PFN is safe, effective and patient friendly device useful for the treatment of all pertrochanteric fractures in post menopausal women age more than 60 years irrespective of their comminution. Early mobilization and rehabilitation is possible since it is closed intramedullary procedure. Weight bearing should be delayed in severely comminuted fractures. It is mandatory to place both proximal screws in the neck, along with distal locking screws for better implant stability in osteoporotic as well as severely comminuted fractures.

\section{References}

1. Boyd HB, Griffin LL. Classification and treatment of trochanteric Fractures. Arch Surgery 1994; 58:853-866.

2. Leung KS. 186 patients, 136 unstable fractures, Randomized Prospective study. JBJS (Br), 1992; 784-B: 
345-51.

3. Banan H, Al Sabti A, Jumulia T, Hart AJ. The treatment of unstable, extracapsular Hip fractures with the $\mathrm{AO} /$ ASIF Proximal Femoral Nail (PFN) - our first 60 cases. Injury. 2002; 33:401-405.

4. Aloyassari G, Langstaff RJ, Jones JWM, Al Lami M. The $\mathrm{AO} /$ ASIF, Proximal Femoral Nail (PFN) for the treatment of unstable trochanteric femoral fracture. Injury. 2002; 33:395-399

5. Kyle Richard F, Cambell Sara J. Intertrochanteric fractures, Chapter-40 Michael W. Chapman, Operative Orthopaedics, 1, 600-603.

6. Nuber S, Ruter A. Stabilization for unstable trochanteric fractures by DHS/PFN Unfail chirurg. 2003; (1060):3947. German

7. Suadan M, Lubbeke A, Sadonstai C, Riand N, Stem R, Ho Hmeyer P. Petrochanteric fractures. Is there an advantage to an intramedullary nail? A randomized prospective study of 206 patients comparing DHS and PFN. J. Oms Trauma. 2002; 16(6):386-393.

8. Mahomed MN, Harrington IJ, Hearn TC. Biomechanical analysis of the Medoff sliding plate. Trauma. 2000; 48(1):93-100.

9. Parken MJ, Hondell HHG. Gamma and other cephalocondylic intramedullary nails Vs extra medullary implants for extra capsular hip fractures.Cochrane library, 2002, 3.

10. Ebraheim N, Mekhail A, Checroun A. Entry point of reconstruction nail, Am J Orthop. 1998; 27:474-476.

11. Seval B, Gavir JM, Gommo JCE, Doblave G. Finite element study of intramedullary osteosynthesis in the treatment of trochanteric fractures of Hip using Gamma nail and PFN. Injury. 2004; 35:130-135.

12. Rutt MS, Krikler SJ, Natie S, Ali MQ. Comparison of DHS and Gamma nail; a prospective, randomized controlled trial Injury. 1995; 26(9):615-618.

13. Simmermacher RK, Bos AM J, Van Dev Werken C. The AO/ ASIF - Proximal Femoral Nail (PFN); a new device for the treatment of unstable proximal femoral fractures. Injury. 1999; 30:327-332.

14. David A, Von Der Heyde D, Pommer A. Therapeutic Possibilities in Trochanteric fractures. Orthopade. 2000; 29(4):294-301.

15. Curter MJ, Jinnah RM, Wilson V. Cunningham. Proximal femoral fractures:A biomechanical study to compare intramedullary fixation. Injury. 1994; 25(2):99-104

16. Rosell PA, Parker MJ. Functional outcome after hip fractures. A 1 year prospective outcome study of 275 patients. Injury. 2003; 34:529-32.

17. Rubuzzi E, Pannonne A, Sehiavetti Santonello D, de Nicalo V, Fancellu G. IMHS Clinical experience in the treatment of petrochanteric fractures. The results of a multicentric Italian study of 981 cases. Injury. 2002: 33:407-412.

18. Seinshimer F. Subtrochanteric fracture of femur JBJS. 1978; 60:300-306.

19. Leung KS. 186 patients, 136 unstable fractures, Randomized Prospective study. JBJS (Br), 1992; 784B:345-51.

20. Banan H, Al Sabti A, Jumulia T, Hart AJ. The treatment of unstable, extracapsular Hip fractures with the $\mathrm{AO} /$ ASIF Proximal Femoral Nail (PFN) - our first 60 cases. Injury 2002; 33:401-405,

21. Aloyassari G, Langstaff RJ, Jones JWM, Al Lami M. The AO/ ASIF, Proximal Femoral Nail (PFN) for the treatment of unstable trochanteric femoral fracture. Injury. 2002; 33:395-399.

22. Teitge RA. Subtrochanteric fractures of femur JBJS (AM) 1976; 58:282

23. Nuber S, Ruter A. Stabilization for unstable trochanteric fractures by DHS/PFN Unfail chirurg. 2003; (1060):3947. German

24. Mahomed MN, Harrington IJ, Hearn TC. Biomechanical analysis of the Medoff sliding plate. Trauma 2000; 48(1):93-100.

25. Parken MJ, Hondell HHG. Gamma and other cephalocondylic intramedullary nails Vs extra medullary implants for extra capsular hip fractures.Cochrane library, 2002, 3.

26. French B, Tornetta P. Use of an interlocked cephalomedullary nail for subtrochanteric fracture stabilization.C1in. Orthop, 1998; 348:95-100

27. Jaylor D, Emelding J. Treatment of comminuted subtrochanteric femoral fractures in young population with a reconstruction nail, Med 1996; 161:735-738

28. Johnson KD. Current techniques in the treatment of subtrochanteric fractures. Technique orthopaedic. 1988; 3:14-24.

29. Henvera A, Domingo LJ. A comparative study of subtrochanteric femoral fractures in a young population with a reconstruction nail. Med. 1996; 161:735-738.

30. Hanson GN, Tullos HS. Subtrochanteric fractures of femur treated with nail plate devices; a retrospective study. Clinical orthopaedics 1978; 131:191-194

31. Velasco RV, Comfort T. Analysis of treatment problems in subtrochanteric fractures of femur $\mathrm{J}$ trauma 1978; 18:513-522

32. Wiss DA, Benn WW. Subtrochanteric fractures of femur. Results of treatment by interlocking nails Clin. Orthop. 1992; 283:232-236

33. WY CL, Shah CH, Lee Z. Subtrochanteric fractures treated with interlocking nailing. J trauma 1991; 31:326333

34. Ramakrishnan M, Prasad SS, Parkinson RW, Kaye JC. Management of subtrochanteric femoral fractures and metastasis using long proximal femoral nail. Injury. 2004; (35):184-190.

35. Schipper B, Steyerberg EW. Treatment of unstable subtrochanteric fractures. Randomized comparison of Gamma Nail and PFNJBJS (Br) 2004; 86(1):86-94.

36. Aronoff PM, Dervis prn J, Widastorm JK. Intra medullary nail fixation as treatment of subtrochanteric fractures of femur J. Trauma. 1971; 1 1:637-650

37. Ebraheim N, Mekhail A, Checroun A. Entry point of reconstruction nail, Am J Orthop. 1998; 27:474-476.

38. Seval B, Gavir JM, CE Gommo J, Doblave G. Finite element study of intramedullary osteosynthesis in the treatment of trochanteric fractures of Hip using Gamma nail and PFN. Injury. 2004; 35:130-135.

39. Rutt MS, Krikler SJ, Natie S, Ali MQ. Comparison of DHS and Gamma nail; a prospective, randomized controlled trial Injury. 1995; 26(9):615-618.

40. Simmermacher RK J, Bos AM, Van Dev Werken C. The AO/ ASIF - Proximal Femoral Nail (PFN); a new device for the treatment of unstable proximal femoral fractures. Injury 1999; 30:327-332.

41. David A, Von Der Heyde D, Pommer A. Therapeutic Possibilities in Trochanteric fractures. Orthopade. 2000; 29(4):294-301. 